\title{
Pathogenic NF1 truncating mutation and copy number alterations in a dedifferentiated liposarcoma with multiple lung metastasis: a case report
}

\author{
Yoon-Seob Kim ${ }^{1,2,3}$, Sun Shin ${ }^{1,2,3}$, Seung-Hyun Jung ${ }^{2,3,4}$ and Yeun-Jun Chung ${ }^{1,2,3,5^{*}}$ (1)
}

\begin{abstract}
Background: Dedifferentiated liposarcoma (DDLPS), which accounts for an estimated 15-20\% of liposarcomas, is a high-grade and aggressive malignant neoplasm, exhibiting a poor response to available therapeutic agents. However, genetic alteration profiles of DDLPS as well as the role of NF1 mutations have not been studied extensively.

Case presentation: The current study reports a patient presenting with rapidly growing DDLPS accompanied by multiple lung and pleural metastases, in whom whole-exome sequencing revealed a NF1 truncating mutation of the known pathogenic variant, c.C7486T, p.R2496X, as well as multiple copy number alterations (CNAs), including the wellknown 12q13-15 amplification, and multiple chromothripsis events encompassing potential cancer-related genes.

Conclusions: Our results suggest that, in addition to the 12q13-15 amplification, NF1 inactivation mutation and other CNAs may contribute to DDLPS tumorigenesis accompanied by aggressive clinical features.
\end{abstract}

Keywords: Case report, Copy number alternation, Liposarcoma, Mutation, NF1, Copy number alteration

\section{Background}

Liposarcoma is the most common type of adult soft tissue sarcoma. Dedifferentiated liposarcoma (DDLPS), which accounts for an estimated $15-20 \%$ of liposarcomas, is a high-grade, aggressive disease that shows a poor response to available therapies [1]. Genetic analysis of DDLPS via The Cancer Genome Atlas (TCGA) project revealed that, compared with other solid tumors, DDLPS carried frequent copy number alterations (CNA) including recurrent amplification at $12 \mathrm{q} 13-15$, and relatively lower somatic mutations with only a few recurrently mutated genes, such as TP53 and ATRX [2]. Regarding CNAs, DDLPS is characterized by

\footnotetext{
* Correspondence: yejun@catholic.ac.kr

'Department of Microbiology, College of Medicine, The Catholic University of Korea, Seoul, Republic of Korea

${ }^{2}$ Integrated Research Center for Genome Polymorphism, College of Medicine, The Catholic University of Korea, Seoul, Republic of Korea

Full list of author information is available at the end of the article
}

highly recurrent amplifications in the $12 \mathrm{q} 13-15$ region that contains the potential oncogenes, MDM2, CDK4, YEATS4, and $F R S 2$, and the adipocytic differentiation factors, DDIT, PTPRQ, and HMGA2 [2-6]. In addition to the 12q13-15 amplification, other CNAs such as the gain of $1 \mathrm{p} 32$ (JUN), and the loss of 17q11 (NF1) and Xq21 (ATRX) have been identified in DDLPS [2-6]. However, genetic alteration profiles of DDLPS have not been studied extensively.

According to studies such as GENIE [7] and TCGA [2], NF1 mutations in DDLPS are rare, being limited to 2/179 cases in GENIE and 1/56 cases in TCGA. (Fig. S1A). MDM4 amplification was observed in 0.6 and $5 \%$ of DDLPS cases via the GENIE study [7] and the TCGA study [2], respectively (Fig. S1A). In TCGA soft tissue sarcoma project $(N=265)$ in cBioPortal database, patients carrying NF1 truncating or missense mutations showed significantly lower NF1 mRNA expression levels than 
those in non-mutated cases. (Mann-Whitney $\mathrm{U}$ test, $P=0.012$ ) (Fig. S1B). Patients carrying MDM4 gains or amplifications showed significantly higher MDM4 mRNA expression levels compared with those of diploid or shallow deletion cases. (Mann-Whitney U test, $P<0.001$ ) (Fig. S1C).

Herein, we report a patient with rapidly growing DDLPS with multiple lung and pleural metastasis, in whom whole-exome sequencing (WES) revealed a NF1 truncating mutation of a known pathogenic variant and multiple CNAs including MDM4 gain.

\section{Case presentation}

An eighty four-year old male visited the outpatient clinic complaining of a painful mass that had been present on his left thigh for 3 years. He was medically healthy and did not have a previous medical or familial history of malignancy. There was no clinical sign of neurofibromatosis. He had twice undergone excisional biopsies (18 and 3 years ago) of the mass at the same location under the presumed diagnosis of lipoma. Preoperative magnetic resonance imaging revealed an approximately $17.5 \times 16.4 \times$ $30.2 \mathrm{~cm}$ sized, extensive, lobulated heterogeneous mass with T2 high, T1 high signal intensity involving the left thigh. A wide local excision was performed. Gross pathology of the tumor showed a well circumscribed tumor mass with internal multi-lobulated areas (Fig. 1a). Multiple lung and pleural metastases were diagnosed via chest computed tomography (Fig. 1b). Histological findings showed dedifferentiated areas with spindle cells without a lipomatous portion and less dedifferentiated areas consisting of round cells with lipomatous portions, along with infiltrated polymorphonuclear cells (Fig. 1c). Based on these clinical and pathological findings, the mass was diagnosed as DDLPS. The patient died 2 months following surgery.

A DDLPS frozen tissue was obtained from the biobank of Seoul St. Mary Hospital (Seoul, Republic of Korea). Genomic DNA was extracted by microdissection of tumor cell rich area ( $>70 \%$ of tumor cell purity) and whole blood of the patient using the DNeasy Blood \& Tissue Kit (Qiagen,

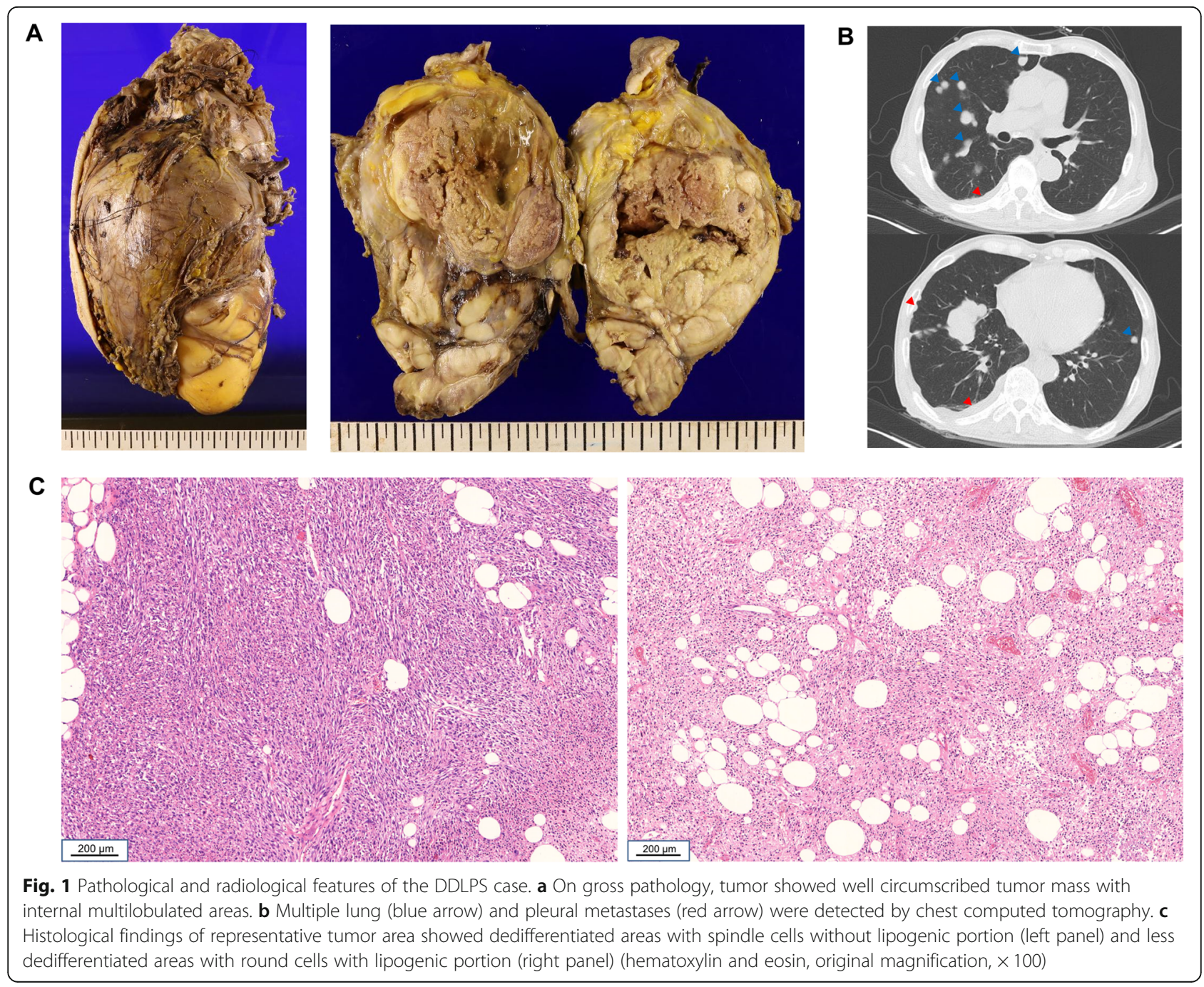


Hilden, Germany). WES was performed using the Agilent SureSelect Human All Exome $50 \mathrm{Mb}$ Kit (Agilent Technologies, Santa Clara, CA) and Illumina HiSeq 2500 platform (Illumina, San Diego, CA). Data pre-processing was done using the best practices workflows of The Genome Analysis toolkit (GATK, v4.1.1) (https://software.broadinsti tute.org/gatk/) to align the sequence reads with the human reference genome (UCSC hg19) and local realignments with base recalibration, and to identify somatic mutations. The web ANNOVAR package was used to select somatic mutations located in the exonic sequences and to predict their functional consequences [8]. In order to obtain reliable and robust mutation calling, the following variants were eliminated: (i) read depth fewer than 20 in either the tumor or matched constitutional tissues; (ii) polymorphisms listed in the population databases of East Asians with a minor allele frequency $0.1 \%$ or more; and (iii) variant allele frequencies less than 5\%. Catalogue of Somatic Mutations in Cancer (COSMIC) mutation signatures were obtained via a Mutalisk package [9] using known mutation signatures of soft-tissue sarcoma [2] (signature 1, 2, 5, and 13). To define CNAs, we used the ngCGH module and

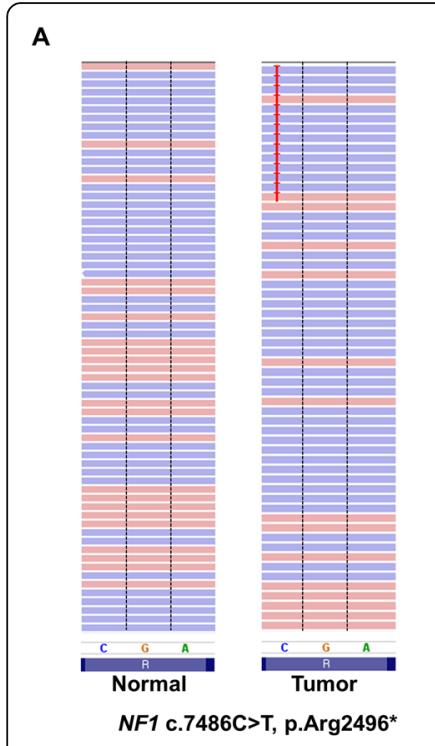

C

Variant allele frequency $15.7 \%$
B

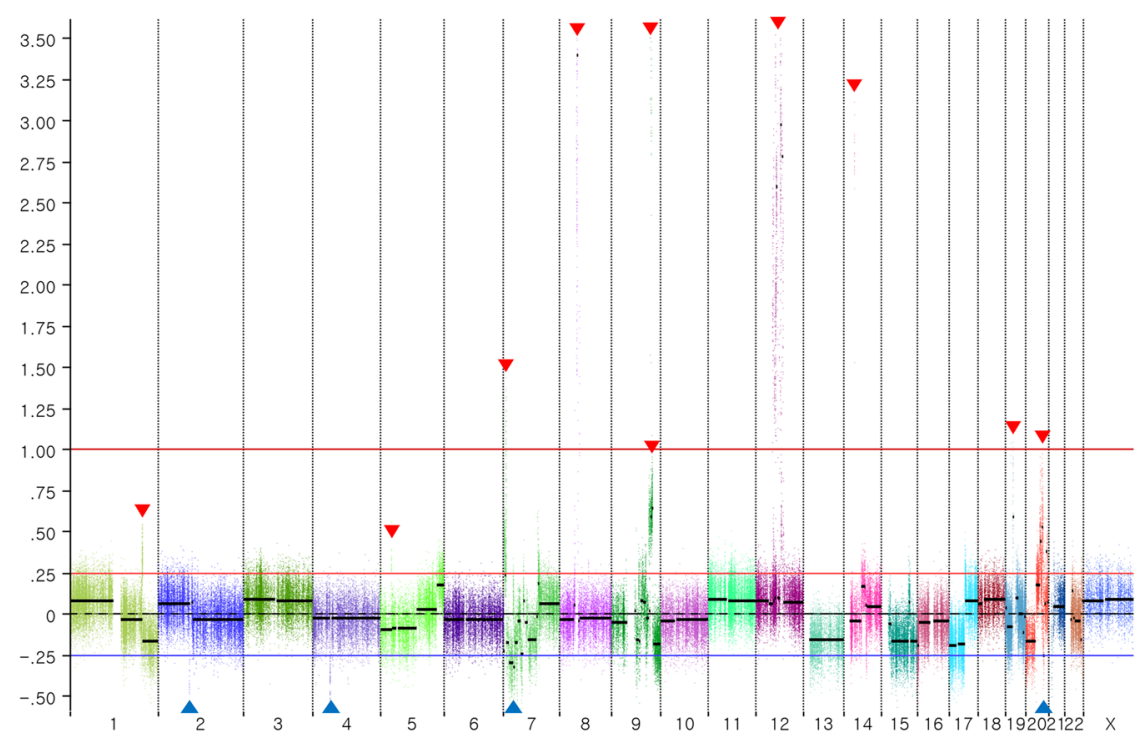

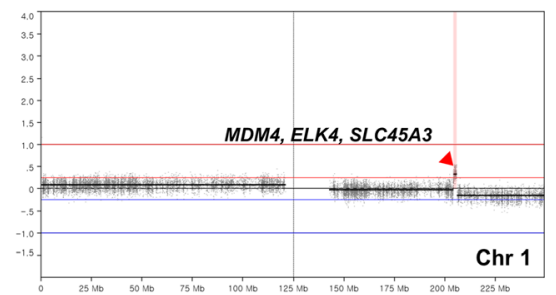
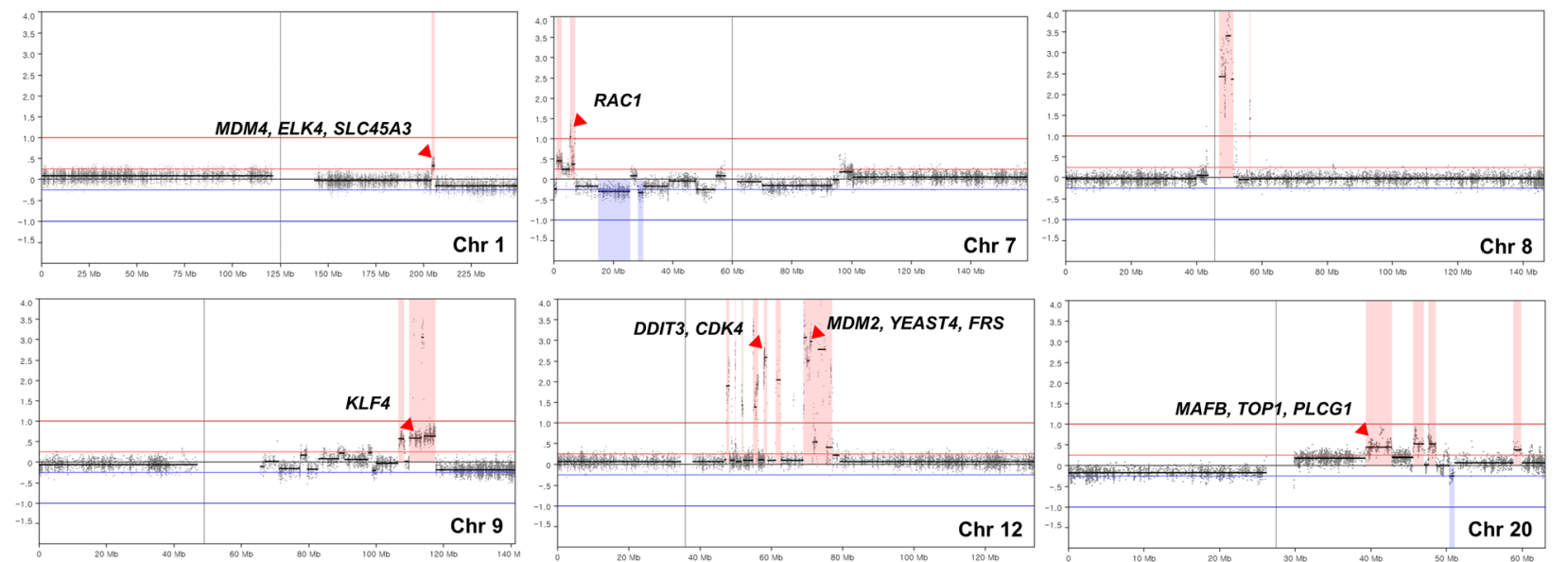

Fig. 2 Identification of genomic alterations by WES from a DDLPS patient. a Identification of a somatic NF1 mutation. The red T letters indicate the presence of a truncating mutation (c.7486C > T, p.Arg2496*, variant allele frequency 15.7\%) in NF1 gene. b Genome-wide copy number alternation profile of the DDLPS patient. X-axis represents individual chromosome, and Y-axis represents depth ratio (tumor/constitutional) in log2 scale. Red and blue lines indicate the threshold of copy gain and copy loss. Bold red line indicates the threshold of amplification. Red and blue arrows represent the copy gain/amplification and loss, respectively. c Copy number alternation profiles of chromosome 1, 7, 8, 9, 12, and 20. Xaxis represents individual chromosome, and Y-axis represents depth ratio (tumor/constitutional) in log2 scale. Red arrows represent the copy gain or amplification regions where known DDLPS related genes are located. Chromothripsis events were observed in 7p, 9q, and 20q 
SNPRank Segmentation statistical algorithm in NEXUS software 9.0 (Biodiscovery, El Segundo, CA). Segments were classified as gains or losses when the $\log 2$ ratio was greater than 0.25 or less than -0.25 , respectively. Amplification was defined as a $\log 2$ ratio greater than 1.0.

The average sequencing depths for tumor and constitutional DNA were 218X and 223X, respectively (Table S1). A total of 36 non-silent mutations were identified in the exonic area (Table S2), which corresponded to a mutation rate of 0.73 per $\mathrm{Mb}$. The NF1 stop gain mutation (c.7486C > T, p.Arg2496*, variant allele frequency 15.7\%) was identified among the cancer-related genes listed in the Cancer Gene Census of COSMIC database (Fig. 2a). This variant was classified as 'pathogenic' in ClinVar DB (RCV000218957.1), and not reported in population level variant databases. There is no germline variant classified as 'pathogenic' or 'likely pathogenic' in ClinVar DB. Thirty-five regions affected by CNAs were found, which correspond to $2 \%$ of the genome, including amplifications on 12q13-15, which included known genes associated with DDLPS pathogenesis, such as DDIT3, CDK4, MDM2, YEATS4, and FRS [2, 4-6] (Figs. 2b \& c; Table S3). In addition to 12q13-15 amplifications, copy gains or amplifications on 1q32, 5p13, 7p22, 8q11-12, 9q31, $12 \mathrm{q} 15-21,14 \mathrm{q} 12,19 \mathrm{p} 12-13$, and $20 \mathrm{q} 12-13$ as well as copy losses on 2p11, 4p11-4q11, 7p21-p14, and 20q13.2 were observed. Several potential cancer-related genes, such as MDM4, ELK4, SLC45A3, RAC1, KLF4, MAFB, TOP1, PLCG1, and PTPRT, were located in the copy gain/ amplification regions (Fig. 2c; Table S3). Chromothripsis events were also observed in 7p, 9q, and 20q (Fig. 2c). In a mutational context, $\mathrm{C}>\mathrm{T}$ mutations were predominant and signature decomposition analysis indicated predominant signatures 1 and 5 (clock-like mutational process) followed by a minor proportion of signatures 2 and 13 (APOBEC-related), which was consistent with the results of a TCGA study on DDLPS [2] (Fig. S2).

\section{Discussion and conclusions}

Although genetic alteration of NF1 is commonly found in liposarcomas $(10-20 \%)[5,6]$, inactivation of NF1 by a mutation or a deletion may contribute to the aggressiveness of liposarcoma $[5,10]$. Processes associated with the occurrence of NF1 mutations in DDLPS remain unclear. Using WES, we identified a pathogenic NF1 truncating mutation with multiple CNAs in a DDLPS case exhibiting aggressive clinical features. The NF1 truncating mutation identified in this case was classified as a 'pathogenic' event (ClinVar) which could act as a driver. In spite of the tumor being located in the extremities, a relatively favorable area [11], the patient presented with aggressive features of a rapidly growing tumor mass accompanied by multiple lung and pleural metastases.
Decreased NF1 expression may lead to dysregulation of the RAS/MAPK pathway, thus contributing to tumorigenesis of the sarcoma [12]. In addition to the known 12q1315 amplification, this case revealed other CNAs where potential cancer related genes, such as $M D M 4$, are located. Amplification of MDM4 (1q32.1) is known to play a synergistic role by inducing the inactivation of TP53 and amplification of $M D M 2$ [13]. Pissaloux et al. reported that a subset of DDLPS exhibited MDM4 amplification as an oncogenic alternative to $M D M 2$ amplification [14]. Chromothripsis events were observed in $7 \mathrm{p}, 9 \mathrm{q}$, and $20 \mathrm{q}$ where potential cancer related genes, such as RAC1, KLF4, MAFB, TOP1, PLCG1, and PTPRT, are located. This result was compatible with that of a previous study which reported that $100 \%$ of liposarcomas (18/18) showed chromothripsis [15].

In conclusion, we report a DDLPS patient who presented with aggressive clinical features. The patient harbored NF1 truncating mutations with multiple CNAs, including the well-known 12q13-15 amplification, and multiple chromothripsis events. Further studies may be needed to elucidate the role of NF1 inactivation mutations and multiple CNAs in DDLPS tumorigenesis accompanied by aggressive clinical features.

\section{Supplementary information}

Supplementary information accompanies this paper at https://doi.org/10. 1186/s12881-020-01137-4.

\section{Additional file 1.}

\section{Abbreviations}

CNA: Copy number alterations; DDLPS: Dedifferentiated liposarcoma; TCGA: The Cancer Genome Atlas; WES: Whole exome sequencing

\section{Acknowledgements}

Not applicable.

\section{Authors' contributions}

YSK and YJC wrote the manuscript. YJC conceived and designed the study. YSK and SS participated in acquisition of data and sample preparation. YSK, SS, and SHJ carried out the molecular genetic studies and following analysis. All authors read and approved the final manuscript.

\section{Funding}

This study was supported by grants from National Research Foundation of Korea (2017R1E1A1A01074913, 2019R1A5A2027588, and

2017M3C9A6047615). The funding body played no role in the design of the study and collection, analysis, and interpretation of data and in writing the manuscript.

\section{Availability of data and materials}

WES data were deposited in the SRA database (Project ID: PRJNA529696). Human reference genome used in this study (UCSC hg19) can be downloaded at the following url: https://hgdownload.soe.ucsc.edu/ downloads.html. Accession numbers of the putative pathogenic variant in this study (NF1 c.7486C > T, p.Arg2496*) in ClinVar database is

RCV000218957.1. Functional annotation of genetic variants listed in Supplementary Table $\mathbf{S} 2$ is generated by the web ANNOVAR package available at the following url: http://wannovar.wglab.org/. Genes listed in The Cancer Gene Census tier 1 curated by COSMIC project (v91 release) is available at the following url: https://cancer.sanger.ac.uk/census. All other 
remaining data are available within the article and in the supplementary data, or available from the authors upon reasonable request.

\section{Ethics approval and consent to participate}

This study was approved by the institutional review board of the Catholic University of Korea (KC15TISI0966)

\section{Consent for publication}

The patient has provided written consent to publish this case report, including medical data and images. A copy of the written consent forms is available for review by the Editor Office of this journal.

\section{Competing interests}

The authors declare that they have no competing interests.

\section{Author details}

'Department of Microbiology, College of Medicine, The Catholic University of Korea, Seoul, Republic of Korea. ${ }^{2}$ Integrated Research Center for Genome Polymorphism, College of Medicine, The Catholic University of Korea, Seoul, Republic of Korea. ${ }^{3}$ Precision Medicine Research Center, College of Medicine, The Catholic University of Korea, Seoul, Republic of Korea. ${ }^{4}$ Department of Biochemistry, The Catholic University of Korea, Seoul, Republic of Korea. ${ }^{5}$ Biomedicine \& Health Sciences, The Catholic University of Korea, Seoul, Republic of Korea.

Received: 28 February 2020 Accepted: 1 October 2020

Published online: 12 October 2020

\section{References}

1. Lee ATJ, Thway K, Huang PH, Jones RL. Clinical and molecular Spectrum of Liposarcoma. J Clin Oncol. 2018:36:151-9.

2. Comprehensive and Integrated Genomic Characterization of Adult Soft Tissue Sarcomas. Cell. 2017;171:950-965.e928. https://pubmed.ncbi.nlm.nih. gov/29100075/.

3. Amin-Mansour A, George S, Sioletic S, Carter SL, Rosenberg M, TaylorWeiner A, et al. Genomic evolutionary patterns of Leiomyosarcoma and Liposarcoma. Clin Cancer Res. 2019:25:5135-42.

4. Beird HC, Wu CC, Ingram DR, Wang WL, Alimohamed A, Gumbs C, et al. Genomic profiling of dedifferentiated liposarcoma compared to matched well-differentiated liposarcoma reveals higher genomic complexity and a common origin. Cold Spring Harb Mol Case Stud. 2018;4. https://pubmed. ncbi.nlm.nih.gov/29610390/.

5. Kanojia D, Nagata Y, Garg M, Lee DH, Sato A, Yoshida K, et al. Genomic landscape of liposarcoma. Oncotarget. 2015:6:42429-44.

6. Barretina J, Taylor BS, Banerji S, Ramos AH, Lagos-Quintana M, Decarolis PL, et al. Subtype-specific genomic alterations define new targets for soft-tissue sarcoma therapy. Nat Genet. 2010;42:715-21.

7. AACR Project GENIE. Powering precision medicine through an international consortium. Cancer Discov. 2017;7:818-31.

8. Chang $X$, Wang K. WANNOVAR: annotating genetic variants for personal genomes via the web. J Med Genet. 2012;49:433-6.

9. Lee J, Lee AJ, Lee JK, Park J, Kwon Y, Park S, et al. Mutalisk: a web-based somatic MUTation AnaLyIS toolKit for genomic, transcriptional and epigenomic signatures. Nucleic Acids Res. 2018;46:W102-w108.

10. Ratner N, Miller SJ. A RASopathy gene commonly mutated in cancer: the neurofibromatosis type 1 tumour suppressor. Nat Rev Cancer. 2015;15:290-301.

11. Dalal KM, Antonescu CR, Singer S. Diagnosis and management of lipomatous tumors. J Surg Oncol. 2008;97:298-313.

12. Philpott C, Tovell H, Frayling IM, Cooper DN, Upadhyaya M. The NF1 somatic mutational landscape in sporadic human cancers. Hum Genomics 2017;11:13.

13. Marine JC, Francoz S, Maetens M, Wahl G, Toledo F, Lozano G. Keeping p53 in check: essential and synergistic functions of Mdm2 and Mdm4. Cell Death Differ. 2006;13:927-34

14. Pissaloux D, Loarer FL, Decouvelaere AV, Paindavoine S, Houlier A, Vernay L, et al. MDM4 amplification in a case of de-differentiated liposarcoma and insilico data supporting an oncogenic event alternative to MDM2 amplification in a subset of cases. Histopathology. 2017:71:1019-23.

15. Cortés-Ciriano I, Lee J-K, Xi R, Jain D, Jung YL, Yang L, et al. Comprehensive analysis of chromothripsis in 2,658 human cancers using whole-genome sequencing. Nat Genet. 2020;52:331-41.

\section{Publisher's Note}

Springer Nature remains neutral with regard to jurisdictional claims in published maps and institutional affiliations.
Ready to submit your research? Choose BMC and benefit from:

- fast, convenient online submission

- thorough peer review by experienced researchers in your field

- rapid publication on acceptance

- support for research data, including large and complex data types

- gold Open Access which fosters wider collaboration and increased citations

- maximum visibility for your research: over $100 \mathrm{M}$ website views per year

At $\mathrm{BMC}$, research is always in progress.

Learn more biomedcentral.com/submissions 